\title{
High optimization process for increasing the attenuation properties of acoustic metamaterials by means of the creation of defects
}

\author{
V. Romero-García, ${ }^{1}$ J. V. Sánchez-Pérez, ${ }^{1, a)}$ L. M. García-Raffi, ${ }^{2}$ J. M. Herrero, ${ }^{3}$ \\ S. García-Nieto, ${ }^{3}$ and X. Blasco ${ }^{3}$ \\ ${ }^{1}$ Centro de Tecnologías Físicas: Acústica, Materiales y Astrofísica, Universidad Politécnica de Valencia, \\ Camino de Vera s/n, 46022 Valencia, Spain \\ ${ }^{2}$ Instituto Universitario de Matemática Pura y Aplicada, Universidad Politécnica de Valencia, \\ Camino de Vera $s / n, 46022$ Valencia, Spain \\ ${ }^{3}$ Department of Systems Engineering and Control, Universidad Politécnica de Valencia, \\ Camino de Vera s/n, 46022 Valencia, Spain
}

(Received 8 July 2008; accepted 12 November 2008; published online 1 December 2008)

\begin{abstract}
An improvement in the attenuation capabilities of acoustic metamaterials by means of the creation of defects is considered here as a multiobjective optimization problem. From this point of view, it is possible to define the optimum strategy in the creation of defects to achieve an important increase in acoustic attenuation in a predetermined range of frequencies. A powerful multiobjective optimization algorithm called evMOGA has been used to solve this problem. The study has been restricted to the case of a two-dimensional sonic crystal formed by rigid cylinders in air, the defects being vacancies in the initial structure. (C) 2008 American Institute of Physics.
\end{abstract}

[DOI: $10.1063 / 1.3040317$ ]

Acoustic metamaterials are currently attracting considerable interest due to the great range of possible applications. In the acoustic case, the term metamaterial involves manmade acoustic media with unusual properties that can be achieved by artificial structurings which are smaller than the length scale of the external stimulus. A great effort has been made in the characterization of these materials. ${ }^{1,2}$ Phononic or sonic crystals (SCs) are considered as a family of acoustic metamaterials defined as a periodic distribution of sound scatterers embedded in another medium with different elastic properties between them. ${ }^{3,4}$ The study of the transmission properties of these metamaterials has attracted increasing attention in recent years due to potential technological applications. ${ }^{5,6}$ Research on increasing the attenuation capability of SCs has been considered a hot topic in the development of acoustic attenuation devices. ${ }^{7,8}$ One of the following options is the creation of vacancies (removing cylinders) in the SC. The use of optimization algorithms as a tool to decide the optimum strategy for removing cylinders has been revealed as an accurate method for improving the attenuation properties of SCs in any range of frequencies, even in a low wavelength regime ${ }^{8}$ where SCs reveal a metamaterialistic behavior. ${ }^{2,6}$ Moreover, the creation of vacancies presents clear advantages - both acoustically and economically-in the design of some filter devices (seemingly a suitable method to design acoustic barriers based on SC which are lighter and cheaper than the classical screens currently in use).

In this paper we analyze various strategies in the creation of vacancies in an initial and complete twodimensional SC formed by rigid cylinders in air, in order to maximize attenuation properties in a predetermined range of frequencies. Our purpose is twofold: on the one hand, to obtain the optimum strategy for the creation of vacancies; on

\footnotetext{
a) Author to whom correspondence should be addressed. Electronic mail: jusanc@fis.upv.es.
}

the other hand, to demonstrate that the optimization method produces a good solution to the presented problem. Our approach consists in the use of a search algorithm based on genetic algorithms, called the epsilon variable multiobjective genetic algorithm ${ }^{9}$ (evMOGA) (also known as $\notin-$ MOGA), in conjunction with the multiple scattering theory (MST) ${ }^{10}$

To optimize the attenuation properties of SCs, we have considered that the attenuation spectra (usually called insertion $\operatorname{loss}^{8}$ ) of the analyzed samples have to fulfill the two following requirements in any chosen optimizing range of frequencies: (i) the average attenuation has to present a maximum and (ii) the standard deviation has to present a minimum. These types of situations where several requirements (termed objective functions) have to be simultaneously improved are known as multiobjective optimization problems and must be solved by simultaneously optimizing the chosen objective functions. ${ }^{11}$ evMOGA, in combination with the MST, is presented as an interesting alternative for solving these types of problems.

In our case, we wish to obtain the configuration of vacancies in an initial SC that (1) maximizes and (2) regularizes the attenuation spectrum in a predetermined range of frequencies. Two objective functions, based on the calculation of the acoustic pressure $p$, are defined to achieve this purpose: the average $\left(J_{1}\right)$ and the standard deviation $\left(J_{2}\right)$ of the acoustic pressure. Mathematically,

$$
\begin{aligned}
& J_{1}(\vec{x})=\bar{p}=\sum_{i}\left|p_{i}(\vec{x})\right| / N_{f}, \\
& J_{2}(\vec{x})=\sqrt{\sum_{i}\left(\left|p_{i}(\vec{x})\right|-\bar{p}\right)^{2} / N_{f}^{2}},
\end{aligned}
$$

where $N_{f}$ represents the number of frequencies considered in the range under study and pairs $\vec{x}=\left(X_{\text {cyl }}, Y_{\text {cyl }}\right)$ provide the position of the existing cylinders in the sample. Minimizing both $J_{1}$ and $J_{2}$ implies obtaining a maximum of the acoustic 

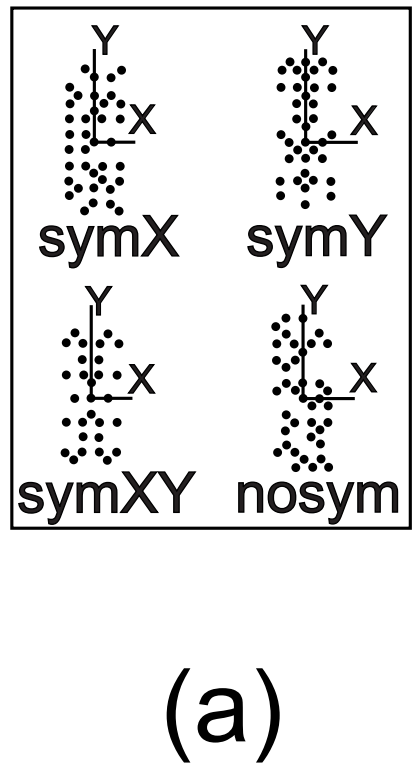

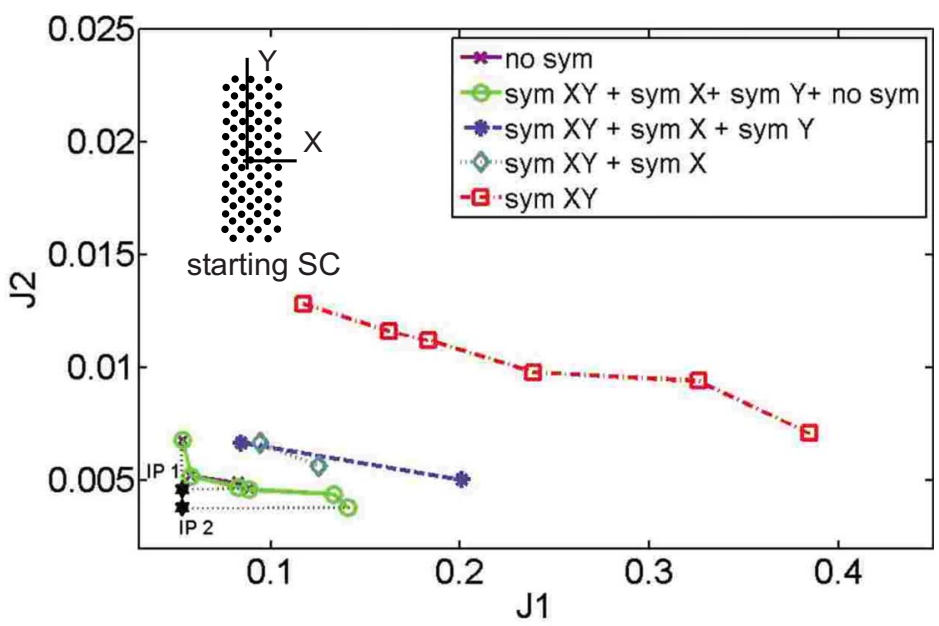

(b)

FIG. 1. (Color online) (a) Examples of the different vacancy creation strategies used. (b) PFs for the mixed method showing each of the steps in the optimization process. The PF for the independent nosym strategy is also represented. The complete and initial SC is also included in the inset.

attenuation and maximizing the flatness of the attenuation spectrum, respectively, both in the range $N_{f}$.

The solutions obtained in a multiobjective optimization process are not unique and they are usually called the Pareto set. The graphical representation of this set is known as the Pareto front ${ }^{12}(\mathrm{PF})$, and it represents the best values, in some sense, of the objective functions considered [Fig. 1(b)]. In general, each dimension of these plots corresponds to the variation in each objective function so, in our case, the corresponding PF is a bidimensional graph where $J_{2}$ is plotted as a function of $J_{1}$. Each point of the PF with coordinates $\left(J_{1 i}, J_{2 i}\right)$ corresponds to an optimized structure.

The method used by evMOGA to obtain a set of optimized solutions consists in searching for the best solutions in the search space by minimizing the objective functions. In general, to find a good solution greatly depends on the optimization path followed. The use of different optimization paths in parallel calculus of the same problem increases the reliability of the results because, if we obtain the same results, this will indicate both the independence of the path followed inside the search space and the proximity to the optimal solution of the problem. Ideal point (IP) from a PF is defined as the point of minimum coordinates $\left(J_{1 \mathrm{~min}}, J_{2 \mathrm{~min}}\right)$. In our case, IP is that one with the best average attenuation and standard deviation, respectively. Distance to the IP is a classical index of quality of the solution in multiobjective optimization problems [see Fig. 1(b)].

In this paper we have defined four strategies in the creation of vacancies (by removing cylinders), taking into account the symmetry axis of the initial SC: (i) symmetry around the $X$ axis $(\operatorname{sym} X)$, (ii) symmetry around the $Y$ axis $(\operatorname{sym} Y)$, (iii) symmetry around both the $X$ and $Y$ axes (sym $X Y$ ), and (iv) random (nosym) [Fig. 1(a)]. The initial SC is formed by 73 cylinders with a $4 \mathrm{~cm}$ diameter, placed in a triangular array with lattice constant $a=6.35 \mathrm{~cm}$. There are seven rows. The acoustic pressure is calculated by means of MST at a point located $1 \mathrm{~m}$ from the middle of the $\mathrm{SC}$ in the direction of $\Gamma X$. The chosen frequency range is from
2300 to $3700 \mathrm{~Hz}$, calculating acoustic pressures each $50 \mathrm{~Hz}$ $\left(N_{f}=29\right)$.

Based on the above symmetries of vacancy generation, multiple execution of evMOGA with different search paths has been performed in this paper. In one search path, we have considered each strategy in the creation of defects in an independent optimization process. The different Pareto sets of each strategy have been independently obtained for choosing the best strategy in the generation of vacancies. To acoustically characterize the results, we have taken into account the following: (1) the IP coordinates and (2) the attenuation area (AA), which is an acoustic parameter defined as the area enclosed between the positive range of spectra and the $0 \mathrm{~dB}$ threshold line in the range of the selected frequencies. This parameter, already used in previous works, ${ }^{8}$ measures the attenuation capability of the considered structure.

In Table I we present the results of the optimization process for the four independently considered strategies and the corresponding values for the initial SC (full structure). $\left(J_{1 \text { min }}, J_{2 \text { min }}\right)$ coordinates represent the coordinates of the IP of the Pareto set for each strategy, $\mathrm{AA}_{\mathrm{av}}$ means the average of the AA for each Pareto set as the considered problem, as explained above, does not have a unique solution. By examining the AA values, we can classify the strategies as follows: $\operatorname{nosym}>\operatorname{sym} Y>\operatorname{sym} X>\operatorname{sym} X Y$. That is, the strategy with the minimum IP (nearest to the origin of the coordinates) presents the maximum AA, i.e., $J_{1}$ and $J_{2}$ are well defined in order to achieve the objective pursued. Moreover,

TABLE I. Optimization values obtained by independently analyzing each of the considered symmetries.

\begin{tabular}{lccc}
\hline \hline & $J_{1 \mathrm{~min}}$ & $J_{2 \text { min }}$ & $\mathrm{AA}_{\mathrm{av}}$ \\
\hline $\operatorname{sym} X Y$ & 0.1263 & 0.0076 & 22406 \\
$\operatorname{sym} X$ & 0.0977 & 0.0052 & 23808 \\
$\operatorname{sym} Y$ & 0.0906 & 0.0054 & 25643 \\
nosym & 0.0530 & 0.0046 & 34464 \\
Full structure & 0.5633 & 0.056 & 11965 \\
\hline \hline
\end{tabular}




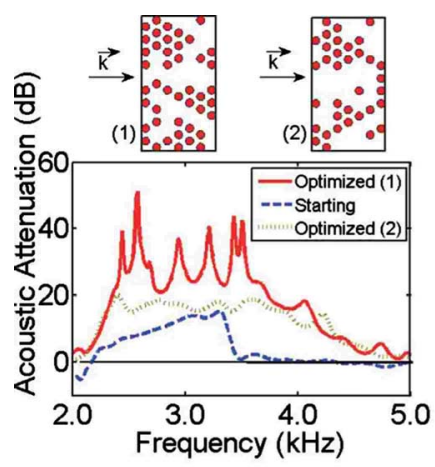

(a)

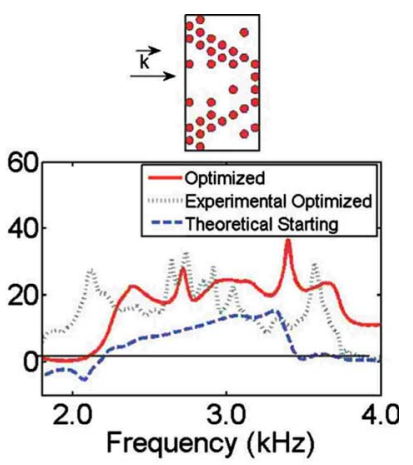

(b)
FIG. 2. (Color online) (a) Attenuation spectra for the nosym samples corresponding to the points that define the IPs of the PFs. The corresponding structures are represented as well. (b) Best nosym structure obtained in the optimization process; theoretical and experimental attenuation spectra are represented. The spectrum of the initial sample is also included.

we can quantitatively obtain an increase in the attenuation capability for each analyzed strategy with respect to the starting structure. This increase is around $200 \%$ in the case of the nosym strategy (see Table I).

To prove that the nosym is not only the optimum strategy but the best, we have developed a strategy for the creation of vacancies, mixing the four strategies defined earlier. In this search path, we have executed 12 runs of the optimization process in four different steps, taking again as an initial population in each run the best individuals obtained in the previous run, and following the order of the strategies obtained in Table I: (i) the three starting run constraints for solutions with $\operatorname{sym} X Y$, (ii) the next three run constraints with only $\operatorname{sym} X$, (iii) the next three with $\operatorname{sym} Y$, and (iv) the final three executions imposing nosym restrictions. In Fig. 1(b) we represent the PFs corresponding to the solutions in each step of this mixed optimization process, plus the PF corresponding to just the nosym strategy. One can see the superposition of both PFs-nosym alone and nosym mixed-and we can conclude that a similar solution is obtained in both cases. This result indicates that the search for the minimum of our problem is independent of the search path, and so the stability of the optimization process is ensured.

Another argument for the selection of the objective functions used in this work is shown in Fig. 2(a). The optimized structures obtained for the points of the PF that define the IP for the nosym case of the mixed strategy [IP2 in Fig. 1(b)] are presented. The left structure [optimized (1)] represents the point corresponding to the PF with the minimum averaged acoustic pressure $J_{1}$ (maximum attenuation level) and maximum standard deviation $\left(J_{2}\right)$. The right-hand example [optimized (2)] represents the structure with maximum $J_{1}$ and minimum $J_{2}$. The corresponding attenuation spectra can also be seen. Both structures show the variation in the attenuation spectra due to the different extreme values of both objective functions considered in the considered PF.
In Fig. 2(b) the best structure obtained in the optimization process and the corresponding attenuation spectra can be seen. This structure corresponds to the point of the nosym PF with the minimum distance to the origin of coordinates. This point means that an agreement between both objective functions is the nearest to the IP. To confirm the validity of the theoretical results obtained, we have drawn the experimental spectra of this optimum structure. We have measured the attenuation spectrum, usually called insertion loss, ${ }^{8}$ analyzing the results with a resolution of $8 \mathrm{~Hz}$. The experiments have been performed in an anechoic chamber using a directional white noise sound source. The good agreement between the theoretical and the experimental spectra can be seen by calculating the corresponding $\mathrm{AA}\left(\mathrm{AA}_{\text {theor }}\right.$ $=31202 \mathrm{~Hz} \mathrm{~dB}$ and $\mathrm{AA}_{\text {expt }}=30215 \mathrm{~Hz} \mathrm{~dB}$ ). Anyway, the differences in the shape between both optimized attenuation spectra (theoretical and experimental) can be explained in terms of the real experimental conditions compared with the ideal conditions considered in the MST model: aluminum cylinders infinitely large, little changes in the length of the experimental lattice constant, acoustic conditions of the anechoic chamber, etc.

As a conclusion, we have analyzed different strategies in the creation of defects (vacancies) in an initial SC, in order to determine which is optimal for achieving the objective of increasing the acoustic attenuation in a predetermined range of frequencies. The nosym strategy is revealed as not only the optimum but also the best among the analyzed strategies. Quantitatively, the resulting structure has doubled the attenuation capability of the initial SC. This result has been proven experimentally and can be extended to other ranges of frequencies (such as ultrasounds) and other types of waves (electromagnetic waves).

This work was partially supported by MEC (Spanish Government) and FEDER funds: Project Nos. 419DPI200507835 and MAT2006-03097 and Generalitat Valenciana Project Nos. GV06/026 and GV/2007/191.

${ }^{1}$ N. Fang, D. Xi, J. Xu, M. Ambati, W. Srituravanich, C. Sun, and X. Zhang, Nature Mater. 5, 452 (2006).

${ }^{2}$ D. Torrent and J. Sánchez-Dehesa, New J. Phys. 10, 023004 (2008).

${ }^{3}$ J. V. Sánchez-Pérez, D. Caballero, R. Martínez-Sala, C. Rubio, J. SánchezDehesa, F. Meseguer, J. Llinares, and F. Gálvez, Phys. Rev. Lett. 80, 5325 (1998).

${ }^{4}$ V. Fokin, M. Ambati, C. Sun, and X. Zhang, Phys. Rev. B 76, 144302 (2007).

${ }^{5}$ J. V. Sánchez-Pérez, C. Rubio, R. Martínez-Sala, R. Sánchez-Grandía, and V. Gómez, Appl. Phys. Lett. 81, 5240 (2002).

${ }^{6}$ D. Torrent and J. Sánchez-Dehesa, New J. Phys. 9, 323 (2007).

${ }^{7}$ W. Kuang, Z. Hou, and Y. Liu, Phys. Lett. A 481, 332 (2004).

${ }^{8}$ V. Romero-García, E. Fuster, L. M. García-Raffi, E. A. Sánchez-Pérez, M. Sopena, J. Llinares, and J. V. Sánchez-Pérez, Appl. Phys. Lett. 88, 174104 (2006).

${ }^{9}$ J. M. Herrero, X. Blasco, M. Martínez, C. Ramos, and J. Sanchis, Biosyst. Eng. 98, 335 (2007).

${ }^{10}$ M. Kafesaki and E. Economou, Phys. Rev. B 60, 11993 (1999).

${ }^{11}$ M. Ehrgott and D. Tenfelde-Podehl, Eur. J. Oper. Res. 151, 119 (2003).

${ }^{12}$ C. Coello, D. Veldhuizen, and G. Lamont, Evolutionary Algorithms for Solving Multi-Objective Problems (Kluwer Academic, Dordrecht, 2002). 